\title{
Integral Field Spectrograph Observations of NGC 4258
}

\author{
Afanasiev V.L. ${ }^{1}$, Courtes G. ${ }^{2}$, Dodonov S.N. ${ }^{1}$, Plana H. ${ }^{2}$ \\ ${ }^{1}$ Special Astrophysical Observatory AS Russia \\ 2 Marseille Observatory, France
}

\begin{abstract}
The nuclear region of the NGC 4258 was observed at the $6 \mathrm{~m}$ Telescope using an integral field spectrograph. Monochromatic maps in $\mathrm{H} \alpha$ and $[\mathrm{NII}]$ 6548/6583 Alines were constructed from the data. Based on monochromatic maps and a velocity field with spatial sampling 0.6 arcsec, and the data published by Courtes et al., (1993) and Cecil et al., (1993) data, we tried to reconstruct the structure of the outflow.
\end{abstract}

\section{Introduction}

The Sbc Hubble type galaxy NGC 4258 (M106) was included by Seyfert (1943) in his list of peculiar galaxies with U-V excess and broad emission lines. After several years discussions (Burbidge \& Burbidge, 1962; Burbidge et al., 1963; Filippenko \& Sargent, 1985; Courtes, 1989; Pecontal et al. 1992) it was established that two systems of broad and narrow lines are superimposed over the nuclear region. The broad component has a linewidth of order of $25 \AA$, narrow line profiles are of order of $5 \AA$. In recent papers Cecil (1991) and Cecil et al. (1992) confirmed the double helical structure foreboded as braided jets and morphological study of NGC 4258 by Courtes et al. (1993) clarifies the general structure of the galaxy.

\section{Observations}

Advantages of integral field spectrograph (IFS) are evident in comparison with long slit spectrographs and could give data with high spatial resolution complementary of those from Fabry-Perot scanning system (TAURUS, Tully et al., 1984; CIGALE, Boulesteix et al., 1986).

Observations were made on May 26, 1992 at the prime focus of the $6 \mathrm{~m}$ Telescope with IFS under seeing conditions 1 to 1.5 arcsec, exposure time was 2 hours. 99 spectra of the object were calibrated using He-Ne-Ar spectral lamp, some exposures of dome light and flat field also were made.

Configuration of IFS which used for the observations was the following:

- Multi-lens array 9x11 squared lenses 2.6 × $2.6 \mathrm{~mm}$ each.

- Several enlargers available for the observatios, they give from 1.6 arsec/lens to $0.15 \mathrm{arsec} / \mathrm{lens}$; we used for the observations enlarger which gives 0.6 arsec/lens. 
North
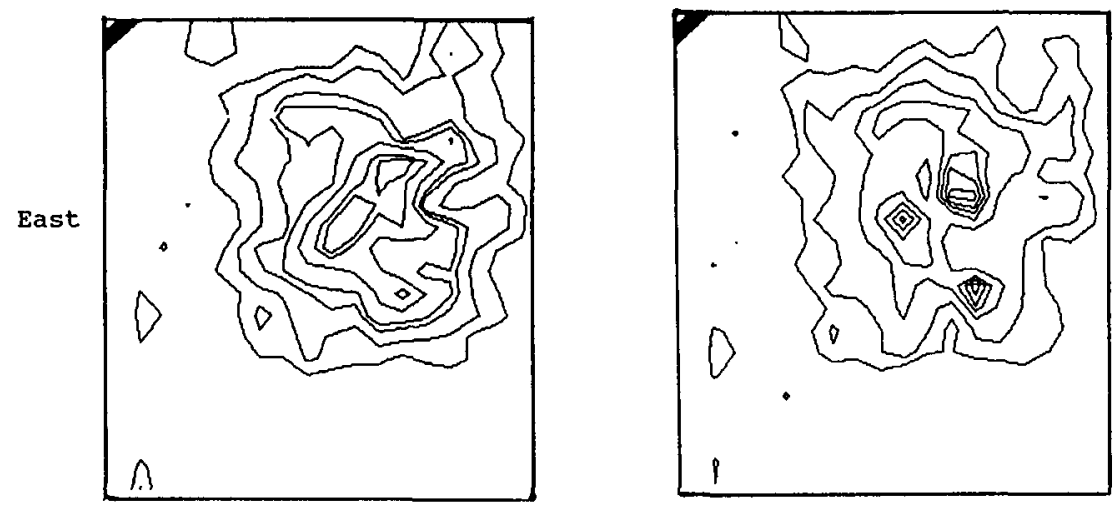

Figure 1. H $\alpha$ (left panel) and [NII] (right panel) maps of the central part of NGC 4258

- Spectral range 6300-6900 $\AA$.

- Spectral resolution $2 \AA / p x$.

The detector was $512 \times 512$ pixels IPCS. Pixel size was $30 \mu \mathrm{m}$. Time resolved imaging mode (TRIM) of observations was used (coordinates of each photon are stored on the hard disk) in order to reduce guiding errors of the telescope and low frequency changes of seeing condition. This mode of observations was first used at CFHT (Nieto et al., 1987) and gave a seeing improvement near $30 \%$. In the case of IFS this method gives the possibility not only of the best seeing selection but also of exact positionning and high quality guiding of the objects during the exposure, most difficult problems for IFS. Data reduction were made using 3D spectroscopic software (Vlasiuk, this conference). Gaussian fits were made for $\mathrm{H} \alpha$ and [NII] lines in order to prepare monochromatic maps and velocity field in each line. Continuum map were built using the complementary spectral range free from emission lines. Accuracy of velocity mesurements is $30 \mathrm{~km} / \mathrm{s}$.

\section{Central region of NGC $\mathbf{4 2 5 8}$}

Monochromatic maps with spatial sampling 0.6 arsec of the central part (5.4 by 6.6 arsec) of NGC 4258 were prepared from $\mathrm{H} \alpha$ and [NII] 6548/6583 $\AA$ lines. They demonstrate us multi-component structures of nuclear region, (Fig. 1).

Continuum map has no peculiarities and gives us possibility to determine the position of the galaxy major axis and photometrical center, (Fig. 2).

$\mathrm{H} \alpha$ velocity field shows rather complicated structure corresponding to multicomponents in nuclear region. Amplitude of this features deviates between $-120 \mathrm{~km} / \mathrm{s}$ and $+180 \mathrm{~km} / \mathrm{s}$ from the systemic velocity.

[NII] velocity field structures repeat the $\mathrm{H} \alpha$ velocity field structure with amplitudes between $-90 \mathrm{~km} / \mathrm{s}$ and $+140 \mathrm{~km} / \mathrm{s}$. 


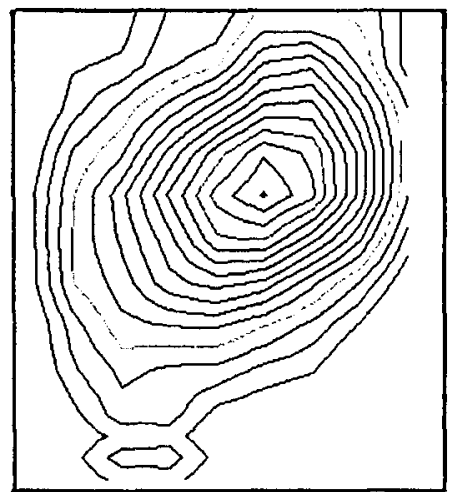

Figure 2. continuum map of the center of NGC 4258

\section{Conclusion}

Data presented above suggest that outflow in NGC 4258 could follow up to the nucleus of the galaxy and in fact we see back side of the outflow in the central part of the galaxy. On the base of data obtaained by Courtes et al. (1993), and Cecil et al. (1992) we attempted to reconstruct the structure of the outflow in NGC 4258, (Fig. 3). More detailed study with higher spectral and spatial resolution observations will be able to clarify physical conditions of this phenomena and gives us understanding of the nature for such effect in the galaxies. Necessity of the spectral resolution $1 \AA$ per pixel comes from the complicated multi component profiles of $\mathrm{H} \alpha$ and more accurate measurements are needed.

\section{References}

Cecil G., Wilson A., Tully B., 1992, ApJ., 390-377.

Courtes G., Petit H., Hua C.T., Martin P.,Blecha A., Huguenin D., Golay M., 1993, A \& A, 268, 419-442. 


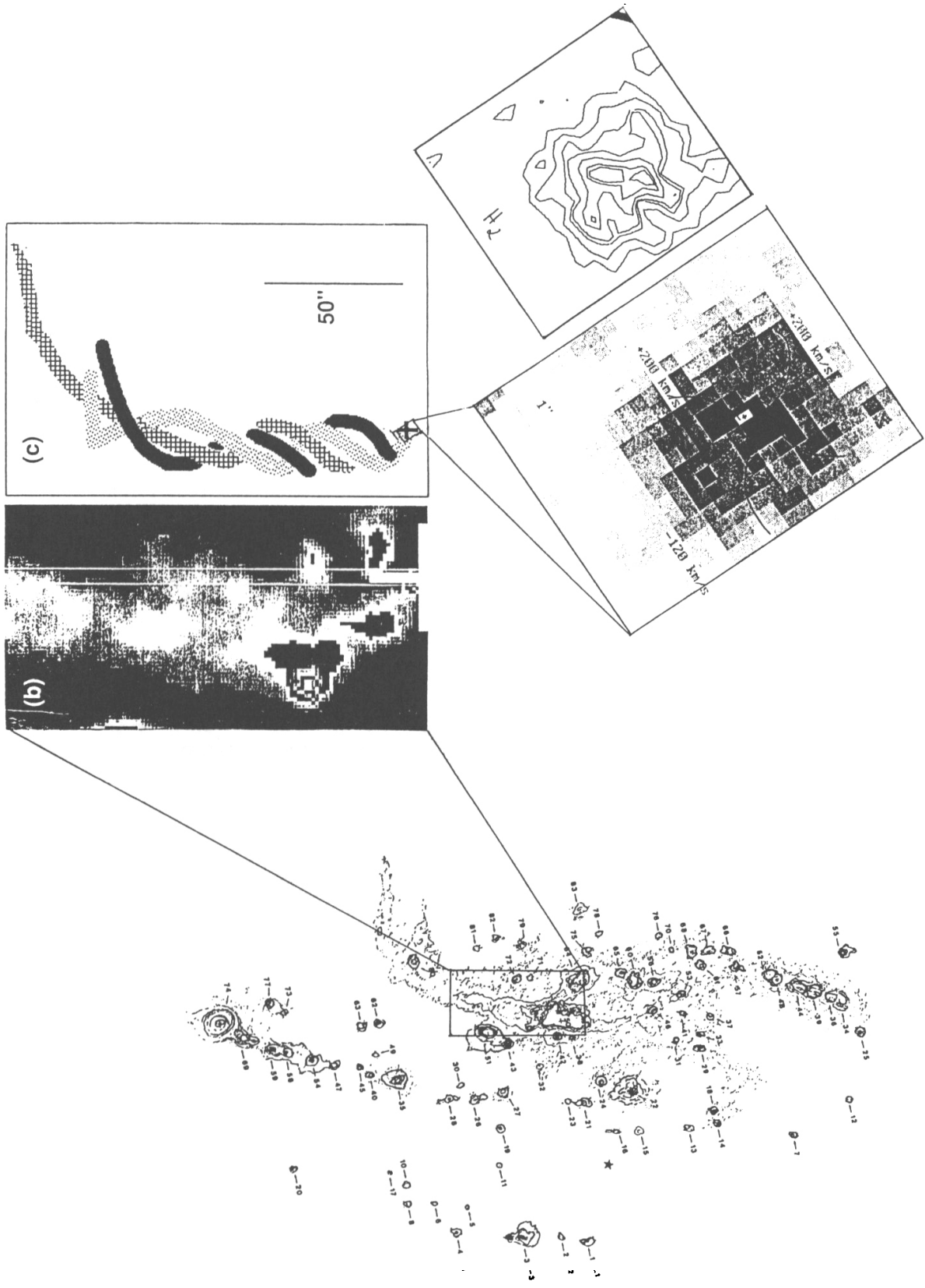

Figure 3. Reconstructed outflow structure from the data of Courtès et al. (1993), Cecil et al. (1992) and from the data obtained with the Multi-Pupil Spectrograph at SAO. 